\title{
ON THE SZEGÖ KERNEL OF AN ANNULUS
}

\author{
SCOTT MCCULLOUGH AND LI-CHIEN SHEN
}

(Communicated by Albert Baernstein II)

\begin{abstract}
In this paper we introduce a class of reproducing kernels of an annulus. Using the properties of the theta functions, we obtain a set of functional equations involving these kernels. We discuss some extremal problems associated with the functional equations.
\end{abstract}

\section{INTRODUCTION}

Let $D$ be a domain on the complex plane bounded by $n$ analytic curves. The Szegö kernel $\hat{K}(z, \zeta)$ of the domain $D$ has the reproducing property

$$
\int_{C} \overline{\hat{K}(z, \zeta)} f(z) d s=f(\zeta), \quad \zeta \in D,
$$

for any function $f$ analytic on $D$ such that

$$
\int_{C}|f(z)|^{2} d s<\infty
$$

where $C$ is the boundary of $D$ and $d s$ the arc length measure. $\hat{K}(z, \zeta)$ is also associated with the following extremal problem: Let $B=\{f: f$ is analytic in $D$ and $|f(z)| \leq 1$ for all $z \in D\}$, and let $\zeta \in D$. Then

$$
\sup _{f \in B}\left|f^{\prime}(\zeta)\right|=2 \pi \hat{K}(\zeta, \zeta)
$$

(see [8, p. 387] for the details of the proof).

The Szegö kernels of the unit disk and the annulus $\mathbb{A}_{q}=\{z: q<|z|<1\}$ are particularly easy to compute. They are, respectively,

$$
\hat{K}(z, w)=\frac{1}{2 \pi} \frac{1}{(1-\bar{w} z)} \quad(|w|,|z|<1)
$$

and

$$
\hat{K}_{q}(z, w)=\frac{1}{2 \pi} \sum_{n=-\infty}^{\infty} \frac{(z \bar{w})^{n}}{1+q^{2 n+1}}, \quad q<|z|,|w|<1
$$

(see [7, p. 391]). We observe that $\hat{K}(z, w)$ satisfies the following simple algebraic identity:

(1) $\hat{K}(z, \lambda) \hat{K}(w, w)-\hat{K}(z, w) \hat{K}(w, \lambda)=\hat{K}(w, w) \overline{\varphi_{w}(\lambda)} \hat{K}(z, \lambda) \varphi_{w}(z)$,

Received by the editors April 30, 1992 and, in revised form, November 6, 1992.

1991 Mathematics Subject Classification. Primary 30C40; Secondary 47A20. 
where $\varphi_{w}(z)=\frac{z-w}{1-w z}$ is a Möbius transformation. This identity arises naturally from the consideration of the Nevanlinna-Pick interpolation problem in the function theory on the unit disk. The main purpose of this note is to generalize the identity (1) for $\hat{K}_{q}(z, w)$. Since the immediate generalization of (1) for $\hat{K}_{q}$ does not exist, we are lead to the consideration of a wider class of kernels

$$
\hat{K}_{q}^{t}(z, w)=\frac{1}{2 \pi} \sum_{h=-\infty}^{\infty} \frac{(z \bar{w})^{n}}{1+t q^{2 n}} .
$$

This collection of kernels arises naturally in the Navanlinna-Pick interpolation problem on the annulus [1] and corresponds to the family of multiplicity one (irreducible in this case) bundle shifts over $\mathbb{A}_{q}$ ([2]; see also [10]). For another "realization" of these kernels and a representation in terms of theta functions see [4]. Clearly, for $t \neq 0$ and $|q|<1$, this series converges for any complex numbers $z, w$ such that $|q|<|z|,|w|<1$, and $\hat{K}_{q}^{q}$ is exactly the Szegö kernel $\hat{K}_{q}$. It is interesting to note that when $t>0$ and $0<q<1, \hat{K}_{q}^{t}$ arises naturally in the following manner. Let $d \mu$ be a measure defined on the boundary $\partial \mathbb{A}_{q}$ of $\mathbb{A}_{q}$ as:

$$
d \mu= \begin{cases}d s & \text { if }|z|=1 \\ \frac{t}{q} d s & \text { if }|z|=q,\end{cases}
$$

where $d s$ is the arc length measure. It is easy to verify that the functions

$$
\phi_{n}(z)=\frac{z^{n}}{\sqrt{2 \pi\left(1+t q^{2 n}\right)}}, \quad n=0, \pm 1, \pm 2, \ldots,
$$

are orthonormal with respect to the measure $d \mu$. Imitating the construction of the Szegö kernel (see [8, p. 389]), we obtain

$$
\sum_{n=-\infty}^{\infty} \phi_{n}(z) \overline{\phi_{n}(w)}=\frac{1}{2 \pi} \sum_{n=-\infty}^{\infty} \frac{(z \bar{w})^{n}}{1+t q^{2 n}}=\hat{K}_{q}^{t}(z, w) .
$$

So $\hat{K}_{q}^{t}$ is obtained by changing the measure of the inner boundary of $\mathbb{A}_{q}$ from the usual arc length measure $d s=q d \theta$ to $\frac{t}{q} d s$; we can therefore regard $\hat{K}_{q}^{t}$ as weighted Szegö kernels.

Since the theta functions will be used throughout this paper, we assume the reader is reasonably familiar with the basic properties of the theta functions.

We begin by recalling

$$
\vartheta_{1}(z)=\vartheta_{1}(z, q)=2 q^{1 / 4} \sin z \prod_{n=1}^{\infty}\left(1-q^{2 n}\right)\left(1-q^{2 n} e^{2 i z}\right)\left(1-q^{2 n} e^{-2 i z}\right),
$$

where $q$ is a complex number such that $0<|q|<1$. It is customary to write $q=e^{\pi i \tau}$ with $\operatorname{Im} \tau>0$. We also introduce

$$
\theta_{1}(x)=-i\left(\sqrt{x}-\frac{1}{\sqrt{x}}\right) q^{1 / 4} \prod_{n=1}^{\infty}\left(1-q^{2 n}\right)\left(1-q^{2 n} x\right)\left(1-q^{2 n} x^{-1}\right) .
$$

Clearly, we have $\vartheta_{1}(z)=\theta_{1}\left(e^{2 i z}\right)$.

To state our main result in full generality, we need to discuss $\hat{K}_{q}^{t}$ in greater detail. To this end, we need to consider the Jordan-Kronecker function

$$
f(\alpha, t)=\sum_{n=-\infty}^{\infty} \frac{\alpha^{n}}{1-t q^{2 n}} .
$$


It is well known that by setting $\alpha=e^{2 i x}$ and $t=e^{2 i y}$

$$
f(\alpha, t)=\frac{i}{2} \vartheta_{1}^{\prime} \frac{\vartheta_{1}(x+y)}{\vartheta_{1}(x) \vartheta_{1}(y)}=\frac{i}{2} \vartheta_{1}^{\prime} \frac{\theta_{1}(\alpha t)}{\theta_{1}(\alpha) \theta_{1}(t)},
$$

where $\vartheta_{1}^{\prime}=\vartheta_{1}^{\prime}(0)$. We note that the series representation for $f(\alpha, t)$ is valid only for $q<|\alpha|<1$; but, since $f(\alpha, t)$ can also be represented in terms of $\vartheta_{1}$ which is entire, it therefore can be analytically continued to the punctured complex plane $0<|\alpha|,|t|<\infty$ and the poles of $f(\alpha, t)$ are located exactly at the zeros of $\theta_{1}$. Clearly, by comparing (2) and (3), we see that

$$
2 \pi \hat{K}_{q}^{t}(z, \lambda)=f(z \bar{\lambda},-t) \text {. }
$$

And from the above remark we will allow the variables $t, \lambda$, and $z$ in $\hat{K}_{q}^{t}$ to be any nonzero complex numbers.

We now state the main result of this paper.

Theorem 1.

$$
\begin{aligned}
& \hat{K}_{q}^{t}(z, \lambda) \hat{K}_{q}^{t}(w, w)-\hat{K}_{q}^{t}(z, w) \hat{K}_{q}^{t}(w, \lambda) \\
& \quad=\hat{K}_{q}^{t}(w, w) \overline{\varphi_{w}(\lambda, q)} \hat{K}_{q}^{s}(z, \lambda) \varphi_{w}(z, q),
\end{aligned}
$$

where $s=t|w|^{2}$ and

$$
\varphi_{w}(z, q)=\frac{w}{|w|} \frac{\theta_{1}(w / z)}{\theta_{1}(\bar{w} z)} .
$$

That the left-hand side of Theorem 1 must yield an expression involving $\hat{K}_{q}^{s}$ for some $s$ can be anticipated from [2], after it has been verified that the minimal normal extension of the subnormal operator multiplication by $z$ on $H_{w}^{2}\left(d \mu_{t}\right)$-the subspace of the Hardy space $H^{2}\left(d \mu_{t}\right)$ consisting of those functions which vanish at $w$-has its spectrum in the boundary of the domain $D$.

It is worthwhile to point out some important properties of $\varphi_{w}(z, q)$. We first note that, from the definition of $\theta_{1}$, it follows easily that

$$
\lim _{q \rightarrow 0} \varphi_{w}(z, q)=\frac{z-w}{1-\bar{w} z} .
$$

So, as $q \rightarrow 0, \varphi_{w}(z, q)$ reduces to a Möbius transformation. More interestingly, $\varphi_{w}(z, q)$ is the conformal map which maps the annulus $\mathbb{A}_{q}$ one-to-one and onto the unit circle with a circular slit in it (see [8, p. 352] for the extremal property associated with it). From this perspective, $\varphi_{w}(z, q)$ can be regarded as a natural analogue of the Möbius transform for the annulus $\mathbb{A}_{q}$. Furthermore, since $\varphi_{w}(w, q)=0$ and

$$
\left|\varphi_{w}(z, q)\right|= \begin{cases}1 & \text { if }|z|=1, \\ |w| & \text { if }|z|=q,\end{cases}
$$

the Green's function $g(z, w)$ for the annulus $\mathbb{A}_{q}$ can be constructed from $\varphi_{w}(z, q)$ as:

$$
g(z, w)=\frac{\ln |w|}{\ln q} \ln |z|-\ln \left|\varphi_{w}(z, q)\right| .
$$

To see that Theorem 1 generalizes the identity (1), we observe that

$$
\hat{K}_{0}^{t}(z, \lambda)=\lim _{q \rightarrow 0} \hat{K}_{q}^{t}(z, \lambda)=\left(\frac{1}{1-z \bar{\lambda}}+\frac{1}{1+t}-1\right),
$$


and together with (6) we obtain

(8) $\hat{K}_{0}^{t}(z, \lambda) \hat{K}_{0}^{t}(w, w)-\hat{K}_{0}^{t}(z, w) \hat{K}_{0}^{t}(w, \lambda)=\hat{K}_{0}^{t}(w, w) \overline{\varphi_{w}(\lambda)} \hat{K}_{o}^{s}(z, \lambda) \varphi_{w}(z)$.

Now (1) follows immediately from (8) by letting $t \rightarrow 0$.

It is interesting to observe that Theorem 1 enables us to solve the following extremal problem:

Let $w, \lambda \in \mathbb{A}_{q}$,

$$
d \mu_{t}= \begin{cases}|d z| & \text { if }|z|=1 \\ \frac{t}{q}|d z| & \text { if }|z|=q\end{cases}
$$

and let $\mathscr{F}=\left\{f: f\right.$ is analytic in $\mathbb{A}_{q}$ such that $f(w)=0$ and $\left.f(\lambda)=1\right\}$.

If $f_{0}(z)=\hat{K}_{q}^{s}(z, \lambda) \varphi_{w}(z, q) / \hat{K}_{q}^{s}(\lambda, \lambda) \varphi_{w}(\lambda, q)$, where $s=t|w|^{2}$, then

$$
\inf _{f \in \mathscr{F}} \int_{\partial \mathbf{A}_{q}}|f(z)|^{2} d \mu_{t}=\frac{1}{\left|\varphi_{w}(\lambda, q)\right|^{2} \hat{K}_{q}^{s}(\lambda, \lambda)}=\int_{\partial \mathbf{A}_{q}}\left|f_{0}(z)\right|^{2} d \mu_{t} .
$$

The proof goes as follows: From Theorem 1 and the Schwarz inequality,

$$
\begin{aligned}
1 & =f(\lambda)=\int_{\partial \mathbf{A}_{q}} \overline{\hat{K}_{q}^{t}(z, \lambda)} f(z) d \mu_{t} \\
& =\int_{\partial \mathbf{A}_{q}}\left(\overline{\hat{K}_{q}^{t}(z, \lambda)}-\frac{\overline{K_{q}^{t}(w, \lambda)}}{\hat{K}_{q}^{t}(w, w)} \overline{\hat{K}_{q}^{t}(z, w)}\right) f(z) d \mu_{t} \\
& =\varphi_{w}(\lambda, q) \int_{\partial \mathbf{A}_{q}} \overline{\hat{K}_{q}^{s}(z, \lambda)} \overline{\varphi_{w}(z, q)} f(z) d \mu_{t} \\
& \leq\left|\varphi_{w}(\lambda, q)\right|\left(\int_{\partial \mathbf{A}_{q}}\left|\hat{K}_{q}^{s}(z, \lambda)\right|^{2}\left|\varphi_{w}(z, q)\right|^{2} d \mu_{t}\right)^{1 / 2}\left(\int_{\partial \mathbf{A}_{q}}|f(z)|^{2} d \mu_{t}\right)^{1 / 2} .
\end{aligned}
$$

Since $\left|\varphi_{w}(z, q)\right|^{2} d \mu_{t}=d \mu_{s}$, we have

$$
\int_{\partial \mathbf{A}_{q}}\left|\hat{K}_{q}^{s}(z, \lambda)\right|^{2}\left|\varphi_{w}(z, q)\right|^{2} d \mu_{t}=\int_{\partial \mathbf{A}_{q}} \overline{\hat{K}_{q}^{s}(z, \lambda)} \hat{K}_{q}^{s}(z, \lambda) d \mu_{s}=\hat{K}_{q}^{s}(\lambda, \lambda) .
$$

Therefore, we conclude

$$
\int_{\partial \mathbf{A}_{q}}|f(z)|^{2} d \mu_{t} \geq \frac{1}{\left|\varphi_{w}(\lambda, q)\right|^{2} \hat{K}_{q}^{s}(\lambda, \lambda)},
$$

and it is easy to see that the equality holds when $f(z)=f_{0}(z)$.

In the following section, we will first prove a key identity involving the products of four theta functions and from which Theorem 1 and three additional corollaries are derived. In $\S 3$ the conjugate kernel $\hat{L}_{q}^{t}$ of $\hat{K}_{q}^{t}$ is introduced via the measure $d \mu$ and we will use one of the above corollaries to establish an identity connecting the Green's function with the product $\hat{K}_{q}^{t} \hat{L}_{q}^{t}$. Further, we show $\mathbb{F}=\hat{K}_{q}^{t} / \hat{L}_{q}^{t}$ solves the extremal problem: Given $\zeta \in \mathbb{A}_{q}$ and $|\zeta|<\alpha<$ $|\zeta| / q$, let $\alpha=q / t$ and $B_{\alpha}=\left\{f\right.$ analytic in $\mathbb{A}_{q} \cup \partial \mathbb{A}_{q}$ and $f(\zeta)=0$ with $|f| \leq 1$ for $|z|=1$ and $|f| \leq \alpha$ for $|z|=q\}$. Then

$$
\sup _{f \in B_{\alpha}}\left|f^{\prime}(\zeta)\right|=\hat{K}_{q}^{t}(\zeta, \zeta)=\mathbb{F}^{\prime}(\zeta) .
$$


Our main results have analogs for fairly general domains of genus $g$. However, in the case of the annulus certain theta function identities are stronger than that obtained in general. For example, we obtain a version of (10) valid only in the Schottky double of $D$ rather than a version of the stronger Lemma 1. Moreover, in the case of the annulus, the results and their proofs are much more concrete and may appeal to a wider audience. Consequently, we leave to a subsequent paper the case of genus $g$, as well as the connections to NevanlinnaPick interpolation and dilation theory.

\section{Proof of TheOREM 1}

In view of (4), it suffices to show that

$$
\begin{aligned}
& f(z \bar{\lambda}, t) f(w \bar{w}, t)-f(z \bar{w}, t) f(w \bar{\lambda}, t) \\
& \quad=f(w \bar{w}, t) \overline{\varphi_{w}(\lambda, q)} f(z \bar{\lambda}, s) \varphi_{w}(z, q) .
\end{aligned}
$$

We now let $z=e^{2 i u}, \lambda=e^{2 i v}, w=e^{2 i a}$, and $t=e^{2 i y}$. Using (4), we can rewrite (9) in terms of $\vartheta_{1}$. It is straightforward (although somewhat tedious) to see that after multiplying both sides through by the common denominator, the identity (9) is equivalent to the following combination of the theta products:

$$
\begin{aligned}
\vartheta_{1}(u-\bar{v}) \vartheta_{1}(a-\bar{a}) \vartheta_{1}(u-\bar{a}+y) \vartheta_{1}(a-\bar{v}+y) \\
\quad-\vartheta_{1}(u-\bar{a}) \vartheta_{1}(a-\bar{v}) \vartheta_{1}(u-\bar{v}+y) \vartheta_{1}(a-\bar{a}+y) \\
=\vartheta_{1}(y) \vartheta_{1}(u-a) \vartheta_{1}(\bar{v}-\bar{a}) \vartheta_{1}(u-\bar{v}+a-\bar{a}+y) .
\end{aligned}
$$

We now prove the above identity by establishing the following slightly more general identity.

\section{Lemma 1.}

$$
\begin{aligned}
\vartheta_{1}(u-b) \vartheta_{1}(a-s) \vartheta_{1}(u-s+y) \vartheta_{1}(a-b+y) \\
\quad-\vartheta_{1}(u-s) \vartheta_{1}(a-b) \vartheta_{1}(u-b+y) \vartheta_{1}(a-s+y) \\
=\vartheta_{1}(y) \vartheta_{1}(u-a) \vartheta_{1}(b-s) \vartheta_{1}(u-b+a-s+y) .
\end{aligned}
$$

We note that the identity (10) is obtained from Lemma 1 by selecting $b=\bar{v}$ and $s=\bar{a}$.

Proof. Let $F(u, a, b, s)$ be the ratio of the quantities on both sides of the above identity with the numerator being the difference of the four theta products and the denominator the product of the four theta functions. We will show that $F \equiv 1$. To this end, we first fix $a, b$, and $s$ and consider $F$ as a function of $u$. We first prove that $F$ is entire. Since $F$ is doubly periodic, it suffices to show that $F$ is analytic at $u=a$ and $u=b-a+s-y$, which are the locations of the possible poles of $F$. We observe that at $u=a$ and $u=b-a+s-y$ the numerator of $F$ is equal to zero, and since the poles of $F$ are all of multiplicity one, $F$ is analytic at $u=a$ and $u=b-a+s-y$. Therefore, $F$ is analtyic in the entire complex plane. From the fact that a doubly periodic entire function is constant, we conclude that $F$ is independent of $u$. Repeating the same arguments for the variables $a, b$, and $s$, we conclude that $F$ is independent of $u, a, b$, and $s$; therefore it is a constant. Now let $a=s$. We see that this constant is 1 . This completes the proof.

The authors are grateful to one of the referees for pointing out that this identity is exactly the identity (45) listed in [6, p. 34] when the genus of the Riemann surface is 1 . 
We now mention an immediate consequence of Lemma 1.

\section{Corollary 1.}

$$
\begin{gathered}
\vartheta_{1}(x) \vartheta_{1}(z) \vartheta_{4}(y) \vartheta_{4}(x+y+z)+\vartheta_{1}(y) \vartheta_{1}(x+y+z) \vartheta_{4}(x) \vartheta_{4}(z) \\
=\vartheta_{4} \vartheta_{1}(x+y) \vartheta_{1}(y+z) \vartheta_{4}(x+z), \quad \text { where } \vartheta_{4}=\vartheta_{4}(0) .
\end{gathered}
$$

Proof. Choosing $y=\frac{\pi \tau}{2}$ in Lemma 1 and using the fact that

$$
\vartheta_{1}\left(z+\frac{\pi \tau}{2}\right)=i q^{-1 / 4} e^{-i z} \vartheta_{4}(z)
$$

we obtain

$$
\begin{gathered}
\vartheta_{1}(u-b) \vartheta_{1}(a-s) \vartheta_{4}(u-s) \vartheta_{4}(a-b)+\vartheta_{1}(s-u) \vartheta_{1}(a-b) \vartheta_{4}(u-b) \vartheta_{4}(a-s) \\
=\vartheta_{4} \vartheta_{1}(u-a) \vartheta_{1}(b-s) \vartheta_{4}(u-b+a-s) .
\end{gathered}
$$

The conclusion now follows by letting $x=u-b, y=s-u$, and $z=a-s$.

The following corollary will be used to derive an identity linking the kernel $\hat{K}_{q}^{t}$ and the Green's function of $\mathbb{A}_{q}$.

\section{Corollary 2.}

$$
\frac{\vartheta_{1}^{\prime}}{\vartheta_{1}}(x)+\frac{\vartheta_{1}^{\prime}}{\vartheta_{1}}(y)+\frac{\vartheta_{1}^{\prime}}{\vartheta_{1}}(z)-\frac{\vartheta_{1}^{\prime}}{\vartheta_{1}}(x+y+z)=\vartheta_{1}^{\prime} \frac{\vartheta_{1}(x+y) \vartheta_{1}(y+z) \vartheta_{1}(x+z)}{\vartheta_{1}(x) \vartheta_{1}(y) \vartheta_{1}(y) \vartheta_{1}(x+y+z)}
$$

Proof. First we appeal to an identity in [11, p. 490]

$$
\frac{\vartheta_{4}^{\prime}}{\vartheta_{4}}(y)+\frac{\vartheta_{4}^{\prime}}{\vartheta_{4}}(z)-\frac{\vartheta_{4}^{\prime}}{\vartheta_{4}}(y+z)=\vartheta_{2} \vartheta_{3} \frac{\vartheta_{1}(y) \vartheta_{1}(z) \vartheta_{1}(y+z)}{\vartheta_{4}(y) \vartheta_{4}(z) \vartheta_{4}(y+z)} \text {. }
$$

We replace $y$ and $z$, respectively, by $y+\frac{\pi \tau}{2}$ and $z+\frac{\pi \tau}{2}$. Using the identities

$$
\vartheta_{4}\left(z+\frac{\pi \tau}{2}\right)=i q^{-1 / 4} e^{-i z} \vartheta_{1}(z)
$$

and

we obtain

$$
\vartheta_{4}(z+\pi \tau)=-q^{-1} e^{-2 i z} \vartheta_{4}(z)
$$

$$
\frac{\vartheta_{1}^{\prime}}{\vartheta_{1}}(y)+\frac{\vartheta_{1}^{\prime}}{\vartheta_{1}}(z)-\frac{\vartheta_{4}^{\prime}}{\vartheta_{4}}(y+z)=\vartheta_{2} \vartheta_{3} \frac{\vartheta_{4}(y) \vartheta_{4}(z) \vartheta_{4}(y+z)}{\vartheta_{1}(y) \vartheta_{1}(z) \vartheta_{1}(y+z)}
$$

In (11), replacing $y$ by $x+y+z$ and $z$ by $-x$ and using the fact that $\vartheta_{1}$ is an odd function, we have

(12) $\frac{\vartheta_{1}^{\prime}}{\vartheta_{1}}(x+y+z)-\frac{\vartheta_{1}^{\prime}}{\vartheta_{1}}(x)-\frac{\vartheta_{4}^{\prime}}{\vartheta_{4}}(y+z)=-\vartheta_{2} \vartheta_{3} \frac{\vartheta_{4}(x+y+z) \vartheta_{4}(x) \vartheta_{4}(y+z)}{\vartheta_{1}(x+y+z) \vartheta_{1}(x) \vartheta_{1}(y+z)}$.

Subtracting (11) from (12), the desired conclusion follows immediately from Corollary 1.

It is worthwhile to point out that, from Corollary 2 , we can easily deduce

$$
f(\alpha, t) f(\beta, t)=t \frac{\partial}{\partial t} f(\alpha \beta, t)-\left(\alpha \frac{\theta_{1}^{\prime}}{\theta_{1}}(\alpha)+\beta \frac{\theta_{1}^{\prime}}{\theta_{1}}(\beta)\right) f(\alpha \beta, t) .
$$

To see this, we let $\alpha=e^{2 i z}, \beta=e^{2 i x}$, and $t=e^{2 i y}$. Recall that $\theta_{1}(\alpha)=$ $\theta_{1}\left(e^{2 i z}\right)=\vartheta_{1}(z)$. Then

$$
\alpha \frac{\theta_{1}^{\prime}}{\theta_{1}}(\alpha)=-\frac{i}{2} \frac{\vartheta_{1}^{\prime}}{\vartheta_{1}}(z)
$$


and

$$
t \frac{\partial f}{\partial t}(\alpha, t)=-\frac{i}{2} \frac{\partial f}{\partial y} .
$$

In (4), replacing $\alpha$ by $\alpha \beta$ and differentiating with respect to $y$, we obtain

$$
t \frac{\partial}{\partial t} f(\alpha \beta, t)=-\left(\frac{i}{2}\right)^{2} \vartheta_{1}^{\prime} \frac{\vartheta_{1}(x+y+z)}{\vartheta_{1}(x+z) \vartheta_{1}(y)}\left(\frac{\vartheta_{1}^{\prime}}{\vartheta_{1}}(x+y+z)-\frac{\vartheta_{1}^{\prime}}{\vartheta_{1}}(y)\right) \text {. }
$$

This and Corollary 2 yield

$$
\begin{aligned}
t \frac{\partial}{\partial t} f & (\alpha \beta, t)-\left(\alpha \frac{\theta_{1}^{\prime}}{\theta_{1}}(\alpha)+\beta \frac{\theta_{1}^{\prime}}{\theta_{1}}(\beta)\right) f(\alpha \beta, t) \\
& =\left(\frac{i}{2}\right)^{2} \vartheta_{1}^{\prime} \frac{\theta_{1}(x+y+z)}{\vartheta_{1}(x+z) \vartheta_{1}(y)}\left[\frac{\vartheta_{1}^{\prime}}{\vartheta_{1}}(x)+\frac{\vartheta_{1}^{\prime}}{\vartheta_{1}}(y)+\frac{\vartheta_{1}^{\prime}}{\vartheta_{1}}(z)-\frac{\vartheta_{1}^{\prime}}{\vartheta_{1}}(x+y+z)\right] \\
& =f(\alpha, t) f(\beta, t) .
\end{aligned}
$$

Now replacing $t$ by $-t$ and choosing $\alpha=z \bar{\lambda}$ and $\beta=w \bar{\zeta}$, we see that the product of weighted Szegö kernels satisfies the following differential equation.

\section{Corollary 3.}

$$
\begin{aligned}
& 2 \pi \hat{K}_{q}^{t}(z, \lambda) \hat{K}_{q}^{t}(w, \zeta) \\
& \quad=t \frac{\partial}{\partial t} \hat{K}_{q}^{t}(z w, \lambda \zeta)-\left(z \bar{\lambda} \frac{\theta_{1}^{\prime}}{\theta_{1}}(z \bar{\lambda})+w \bar{\zeta} \frac{\theta_{1}^{\prime}}{\theta_{1}}(w \bar{\zeta})\right) \hat{K}_{q}^{t}(z w, \lambda \zeta)
\end{aligned}
$$

\section{A Relation betWeEN $\hat{K}_{q}^{t}$ AND the GREen's FUnCtion}

Let $g(z, \zeta)$ be the Green's function of the domain $D$ bounded by $n$ analytic curves, and let $h(z, \zeta)$ be a harmonic conjugate of $g(z, \zeta)$. Here we must bear in mind that $h(z, \zeta)$ is not a single-valued function of $D$. We let

$$
p(z, \zeta)=g(z, \zeta)+i h(z, \zeta)
$$

Since $h(z, \zeta)$ is not single-valued, $p(z, \zeta)$ is a multiple-valued analytic function on $D$. One of the most important properties of $p(z, \zeta)$ is that when $z \in \partial D$ (the boundary of $D$ ),

$$
\frac{1}{i} p^{\prime}(z, \zeta) d z=\frac{\partial g}{\partial n}(z, \zeta) d s ;
$$

that is, when $z \in \partial D, \frac{1}{i} p^{\prime}(z, \zeta) d z$ is precisely the Poisson kernel for the domain $D$ (see p. 305 of [8]). Let $\hat{L}(z, \zeta)$ be the conjugate kernel of $\hat{K}(z, \zeta)$. We mention the following interesting relation between $\hat{K}, \hat{L}$, and $p^{\prime}(z, \zeta)$ :

$$
2 \pi \frac{\hat{K}(z, \zeta) \hat{L}(z, \zeta)}{\hat{K}(\zeta, \zeta)}=-p^{\prime}(z, \zeta)+\sum_{k=1}^{n-1} \beta_{k} w_{k}^{\prime}(z)
$$

(see [8, p. 391]).

We will confine ourselves to the domain $\mathbb{A}_{q}, 0<q<1$. In this case the summation in (14) has just one term, and moreover,

$$
w^{\prime}(z)=\frac{1}{z \ln q} \text {. }
$$


So (14) becomes

$$
2 \pi \frac{\hat{K}_{q}(z, \zeta) \hat{L}_{q}(z, \zeta)}{\hat{K}_{q}(\zeta, \zeta)}=-p^{\prime}(z, \zeta)+\frac{\beta}{\ln q} \frac{1}{z}
$$

for some constant $\beta$.

We now extend the identity (15) to the weighted Szegö kernel. To this end, we define (see [8, p. 390])

$$
\hat{L}_{q}^{t}(z, \zeta)=\frac{1}{2 \pi(z-\zeta)}+\frac{1}{2 \pi} \int_{\partial \mathbf{A}_{q}} \frac{\hat{K}_{q}^{t}(\zeta, w)}{w-z} d \mu .
$$

When $t$ is real, it is easy to see that

$$
z \hat{L}_{q}^{t}(z, \zeta)=\overline{\hat{K}_{q}^{t}\left(\frac{1}{\bar{z}}, \zeta\right)}=\hat{K}_{q}^{t}\left(\frac{1}{z}, \bar{\zeta}\right) .
$$

We state the following generalization of (15).

Theorem 2. Let $t$ be real and $0<q<1$. Then

$$
\frac{2 \pi \hat{K}_{q}^{t}(z, \zeta) \hat{L}_{q}^{t}(z, \zeta)}{\hat{K}_{q}^{t}(\zeta, \zeta)}=-p^{\prime}(z, \zeta)+\frac{1}{t \bar{\zeta}} p^{\prime}\left(\frac{1}{t \bar{\zeta}}, \zeta\right) \frac{1}{z} .
$$

Proof. We first express $p^{\prime}(z, \zeta)$ in terms of the theta functions. From (7) we see that

$$
\begin{aligned}
p^{\prime}(z, \zeta) & =\frac{\ln |\zeta|}{\ln q} \frac{1}{z}-\frac{\varphi_{\zeta}^{\prime}(z, q)}{\varphi_{\zeta}(z, q)} \\
& =\left(\frac{\ln |\zeta|}{\ln q}+\frac{\zeta}{z} \frac{\theta_{1}^{\prime}}{\theta_{1}}\left(\frac{\zeta}{z}\right)+z \bar{\zeta} \frac{\theta_{1}^{\prime}}{\theta_{1}}(z \bar{\zeta})\right) \frac{1}{z}
\end{aligned}
$$

or

$$
z p^{\prime}(z, \zeta)=\frac{\ln |\zeta|}{\ln q}+\frac{1}{2 i} \frac{\vartheta_{1}^{\prime}}{\vartheta_{1}}(v-u)+\frac{1}{2 i} \frac{\vartheta_{1}^{\prime}}{\vartheta_{1}}(u-\bar{v}),
$$

where $z=e^{2 i u}$ and $\zeta=e^{2 i v}$. Here we use the fact that $\vartheta_{1}^{\prime}(u)=2 i z \theta_{1}^{\prime}(z)$. In (18) we now choose $z=\frac{1}{t \xi}=e^{2 i(v-y)} \quad\left(t=e^{2 i y}\right)$. Then

$$
\frac{1}{t \bar{\zeta}} p^{\prime}\left(\frac{1}{t \bar{\zeta}}, \zeta\right)=\frac{\ln |\zeta|}{\ln q}+\frac{1}{2 i} \frac{\vartheta_{1}^{\prime}}{\vartheta_{1}}(v-\bar{v}+y)-\frac{1}{2 i} \frac{\vartheta_{1}^{\prime}}{\vartheta_{1}}(y) .
$$

Hence, from (18), (19), and Corollary 2, we have

$$
\begin{aligned}
& -z p^{\prime}(z, \zeta)+\frac{1}{t \bar{\zeta}} p^{\prime}\left(\frac{1}{t \bar{\zeta}}, \zeta\right) \\
& \quad=\frac{i}{2}\left(\frac{\vartheta_{1}^{\prime}}{\vartheta_{1}}(v-u)+\frac{\vartheta_{1}^{\prime}}{\vartheta_{1}}(u-\bar{v})+\frac{\vartheta_{1}^{\prime}}{\vartheta_{1}}(y)-\frac{\vartheta_{1}^{\prime}}{\vartheta_{1}}(v-\bar{v}+y)\right) \\
& =i \frac{\vartheta_{1}^{\prime}}{2} \frac{\vartheta_{1}(v-\bar{v}) \vartheta_{1}(v-u+y) \vartheta_{1}(u-\bar{v}+y)}{\vartheta_{1}(v-u) \vartheta_{1}(u-\bar{v}) \vartheta_{1}(y) \vartheta_{1}(v-\bar{v}+y)} .
\end{aligned}
$$

On the other hand, from (5) and (16), we have

$$
\begin{aligned}
\frac{2 \pi z \hat{K}_{q}^{-t}(z, \zeta) \hat{L}_{q}^{-t}(z, \zeta)}{\hat{K}_{q}^{-t}(\zeta, \zeta)} & =\frac{z f(z \bar{\zeta}, t) f\left(\frac{\zeta}{z}, t\right)}{f(\zeta \bar{\zeta}, t)} \\
& =\frac{i}{2} \vartheta_{1}^{\prime} \frac{\vartheta_{1}(u-\bar{v}+y) \vartheta_{1}(v-u+y) \vartheta_{1}(v-\bar{v})}{\vartheta_{1}(y) \vartheta_{1}(u-\bar{v}) \vartheta_{1}(v-u) \vartheta_{1}(v-\bar{v}+y)}
\end{aligned}
$$


Now, replacing $-t$ by $t$, the desired conclusion follows immediately from (20), (21), and the observation that

$$
\frac{1}{t \bar{\zeta}} p^{\prime}\left(\frac{1}{t \bar{\zeta}}, \zeta\right)=-\frac{1}{t \bar{\zeta}} p^{\prime}\left(-\frac{1}{t \bar{\zeta}}, \zeta\right)
$$

Theorem 3. Let $t$ be real and $0<q<1$. Then, for $z \in \partial \mathbb{A}_{q}$,

$$
\overline{\hat{K}_{q}^{t}(z, \zeta)} d \mu=\frac{1}{i} \hat{L}_{q}^{t}(z, \zeta) d z .
$$

Proof. We first recall that

$$
\theta_{1}\left(\frac{z}{q^{2}}\right)=-\frac{z}{q} \theta_{1}(z)
$$

We now verify the identity (22) for $|z|=q$. Then $1 / z=\bar{z} / q^{2}$ and $d z / z=$ $-i d s / q$. The minus sign is attributable to the fact that the orientation of the line integral in the inner boundary is clockwise. Then from (16) and (23), we have

$$
\begin{aligned}
\frac{2 \pi}{i} \hat{L}_{q}^{t}(z, \zeta) d z & =\frac{1}{i} f\left(\frac{\zeta}{z},-t\right) \frac{d z}{z}=-\frac{i}{2} \vartheta_{1}^{\prime} \frac{\theta_{1}(-\zeta t / z)}{\theta_{1}(-t) \theta_{1}(\zeta / z)} \frac{d s}{q} \\
& =-\frac{i}{2} \vartheta_{1}^{\prime} \frac{\theta_{1}\left(-\bar{z} \zeta t / q^{2}\right)}{\theta_{1}(-t) \theta_{1}\left(\zeta \bar{z} / q^{2}\right)} \frac{d s}{q} \\
& =-\frac{i}{2} \vartheta_{1}^{\prime} \frac{\theta_{1}(-\zeta \bar{z} t)}{\theta_{1}(-t) \theta_{1}(\bar{z} \zeta)} \frac{t}{q} d s=2 \pi \overline{\hat{K}_{q}^{t}(z, \zeta)} d \mu .
\end{aligned}
$$

The proof for $|z|=1$ is similar; we therefore omit it here.

The identity (22) enables us to solve the following extremal problem: Given $\zeta \in \mathbb{A}_{q}$ and $|\zeta|<\alpha<\frac{|\zeta|}{q}$, let

$$
\begin{aligned}
B_{\alpha}=\{f: f & \text { is analytic in } \mathbb{A}_{q} \cup \partial \mathbb{A}_{q} \text { and } f(\zeta)=0 \\
& \text { such that }|f(z)| \leq 1 \text { if }|z|=1 \text { and }|f(z)| \leq \alpha \text { if }|z|=q\} .
\end{aligned}
$$

Then

$$
\sup _{f \in B_{\alpha}}\left|f^{\prime}(\zeta)\right|=2 \pi \hat{K}_{q}^{t}(\zeta, \zeta), \quad \text { where } t=q / \alpha .
$$

To see this, let $\mathbb{F}(z)=\hat{K}_{q}^{t}(z, \zeta) / \hat{L}_{q}^{t}(z, \zeta)$. We should remark here that the reason, for a given $\zeta \in \mathbb{A}_{q}$, that the value of $\alpha$ is restricted between $|\zeta|$ and $\frac{|\zeta|}{q}$ is to prevent the zeros of $\hat{L}_{q}^{t}(z, \zeta)$ in $\mathbb{A}_{q}$, so $\mathbb{F}(z)$ can be analytic in $\mathbb{A}_{q}$. From (22) we observe that

$$
|\mathbb{F}(z)|= \begin{cases}1 & \text { if }|z|=1 \\ \alpha & \text { if }|z|=q\end{cases}
$$

moreover, for $z \in \partial \mathbb{A}_{q}$,

$$
\begin{aligned}
\frac{1}{i} \mathbb{F}(z)\left(\hat{L}_{q}^{t}(z, \zeta)\right) d z & =\frac{1}{i} \hat{K}_{q}^{t}(z, \zeta) \hat{L}_{q}^{t}(z, \zeta) d z \\
& =\hat{K}_{q}^{t}(z, \zeta) \overline{\hat{K}_{q}^{t}(z, \zeta)} d \mu>0 .
\end{aligned}
$$


We also note that $\hat{L}_{q}^{t}(z, \zeta)$ has exactly one pole at $z=\zeta$ and

$$
\hat{L}_{q}^{t}(z, \zeta)=\frac{1}{2 \pi} \frac{1}{z-\zeta}+g(z)
$$

where $g(z)$ is analtytic in $\mathbb{A}_{q}$ and the power series expansion of $g(z)$ at $z=\zeta$ is

$$
g(z)=c_{0}+c_{1}(z-\zeta)+\cdots, \quad \text { where } c_{0}=\frac{t}{\zeta} \frac{\theta_{1}^{\prime}(-t)}{\theta_{1}(-t)}-\frac{1}{2 \zeta} .
$$

Therefore, from (24)-(27) and the fact that $f(\zeta)=0$,

$$
\begin{aligned}
\left|f^{\prime}(\zeta)\right| & =\left|f^{\prime}(\zeta)+2 c_{0} f(\zeta)\right|=\left|\frac{2 \pi}{i} \int_{\partial \mathbf{A}_{q}}\left(\hat{L}_{q}^{t}(z, \zeta)\right)^{2} f(z) d z\right| \\
& \leq 2 \pi \int_{\partial \mathbf{A}_{q}}\left|\left(\hat{L}_{q}^{t}(z, \zeta)\right)^{2} \mathbb{F}(z) d z\right| \\
& =\frac{2 \pi}{i} \int_{\partial \mathbf{A}_{q}}\left(\hat{L}_{q}^{t}(z, \zeta)\right)^{2} \mathbb{F}(z) d z=\mathbb{F}^{\prime}(\zeta) \\
& =\frac{2 \pi}{i} \int_{\partial \mathbf{A}_{q}} \hat{K}_{q}^{t}(z, \zeta) \hat{L}_{q}^{t}(z, \zeta) d z=2 \pi \hat{K}_{q}^{t}(\zeta, \zeta) .
\end{aligned}
$$

We point out here that when $t=q, c_{0}=0$; therefore, the requirement that $f(\zeta)=0$ is not needed in this case. We also remark that since $\mathbb{F}(z)$ is the ratio of theta functions, it is quasi-periodic, so the restriction on $\alpha$ can be relaxed to $q^{2 n}|\zeta|<\alpha<|\zeta| q^{2 n-1}$ for $n=0, \pm 1, \pm 2, \ldots$. But, for $q^{2 n+1}|\zeta| \leq$ $\alpha \leq q^{2 n}|\zeta|, \hat{L}_{q}^{t}(z, \zeta)$ has one zero in $\mathbb{A}_{q}$, so in this case the extremal value $2 \pi \hat{K}_{q}^{t}(\zeta, \zeta)$ is achieved by the function $\mathbb{F}(z)$ which is meromorphic in $\mathbb{A}_{q} U$ $\partial \mathbf{A}_{q}$.

We end this paper by remarking that in [6, pp. 130-133], by expressing the Szegö kernel in terms of the theta functions on Riemann surfaces, Fay also investigated this extremal problem for multiply connected domain. The Szegö kernel he considered arises from the usual arc length measure on each of the boundary components, whereas in this paper the family of Szegö kernels for an annulus are constructed from the weighted arc length measure on the boundary. It is interesting to note the effect of the weight on the location of the zeros of the kernels. From the standpoint of this paper, it is natural to devote further investigation to the kernels associated to the weighted arc length measure for the multiply connected domains.

\section{REFERENCES}

1. M. B. Abrahamse, The Pick interpolation theorem for finitely connected domains, Michigan Math. J. 26 (1979), 195-203.

2. M. B. Abrahamse and R. G. Douglas, A class of subnormal operators related to a multiply connected domain, Adv. Math. 121 (1976), 106-108.

3. J. Agler, Rational dilation on an annulus, Ann. of Math. (2) 121 (1985), 537-564.

4. J. A. Ball and K. F. Clancey, Reproducing kernels for Hardy spaces on multiply connected domains, preprint.

5. S. Bergman, The kernel function and conformal mapping, Amer. Math. Soc. Survey 5 (1950).

6. John D. Fay, Theta functions on Riemann surfaces, Lecture Notes in Math., vol. 352, Springer, New York, 1973, p. 352. 
7. G. Misra, Curvative inequalities and extremal properties of bundle shifts, J. Operator Theory 11 (1984), 305-318.

8. Z. Nehari, Conformal mapping, Dover, New York, 1985.

9. V. I. Paulsen, Completely bounded maps and dilations, Pitman Press, New York, 1986.

10. D. Sarason, The $H^{p}$ spaces of an annulus, Mem. Amer. Math. Soc., vol. 56, Amer. Math. Soc., Providence, RI, 1975.

11. E. T. Whittaker and G. N. Watson, A course of modern analysis, 4th ed., Cambridge Univ. Press, New York, 1958.

Department of Mathematics, University of Florida, Gainesville, Florida 32611

E-mail address, S. McCullough: sam@math. uf l.edu

E-mail address, Li-Chien Shen: shen@math.ufl.edu 